\title{
Efficiency of Cell Therapy to GC-Induced ONFH: BMSCs with Dkk-1 Interference Is Not Superior to Unmodified BMSCs
}

\author{
Wei Zhun (D), Li Donghai $(\mathbb{D}$, Yang Zhouyuan, Zhao Haiyan $(\mathbb{D}$, and Kang Pengde (i) \\ Department of Orthopaedics Surgery, West China Hospital, Sichuan University, No. 37 Wainan Guoxue Road, Chengdu, China \\ Correspondence should be addressed to Kang Pengde; kangpengde@163.com
}

Received 19 December 2017; Revised 8 March 2018; Accepted 5 April 2018; Published 22 May 2018

Academic Editor: Huseyin Sumer

Copyright (c) 2018 Wei Zhun et al. This is an open access article distributed under the Creative Commons Attribution License, which permits unrestricted use, distribution, and reproduction in any medium, provided the original work is properly cited.

\begin{abstract}
Glucocorticoid-induced osteonecrosis of the femoral head (ONFH) is a hip disorder, and it threatens patients who require megadose of steroid therapies. Nowadays, no valid therapies can reverse the development of GC-induced ONFH once it occurs. Stem cell therapy to GC-induced ONFH would be a promising choice. Although the pathogenesis of GC-induced ONFH is not yet fully clear, Dickkopf-1 (Dkk-1) upregulated by excessive GC use, which hinders the canonical Wnt pathway, could be an explanation. Thus, the aim of the present work lies in investigating the efficiency of the allograft bone marrow stem cells (BMSCs) with Dkk-1 interference in preventing the progression of the GC-induced ONFH. Lentivirus-meditated Dkk-1 RNAi was introduced into BMSCs which was exposed to dexamethasone $\left(10^{-6} \mathrm{~mol} / \mathrm{L}\right)$ in vitro. This interference blocked Dkk-1 overexpression by GC and afterwards prompted the transduction of $\mathrm{Wnt} / \beta$-catenin in which the Runx 2 and PPAR $\gamma$ were upregulated and downregulated, respectively. Thus, the osteogenesis was promoted while adipogenesis was inhibited. In vivo, GC-induced ONFH rats were treated by allotransplantation of BMSCs with Dkk-1 interference, and the progression of the disease was prevented. However, the effects were not significantly superior to treatment with nongenetically modified or normal BMSCs.
\end{abstract}

\section{Introduction}

Excessive glucocorticoid (GC) use is one of the etiologies of osteonecrosis of the femoral head (ONFH). Although the pathophysiological mechanism of GC-induced ONFH remains unclear, reduced osteogenesis due to an aberrant decrease in the bone marrow mesenchymal stem cell (BMSC) pool may play a role $[1,2]$. Thus, in addition to traditional procedures (e.g., core decompression, osteotomies, and bone grafting), BMSC implantation has been proposed as a potential therapy for GC-induced ONFH, especially in the early stages, with the aim of delaying or even obviating total hip arthroplasty (THA) [3-7].

BMSC implantation is often combined with core decompression. BMSC implantation involves the following steps $[8,9]$ : (1) harvesting of autologous or allogeneic BMSCs; (2) canonical core decompression; and (3) implantation of BMSCs in necrotic areas through the decompression tunnel. Although improved outcomes have been observed for
BMSC treatment compared with canonical core decompression, concerns about the remaining osteogenic capacity or proliferation of BMSCs in the repair of GC-induced ONFH [10-12] have limited the widespread use of this treatment.

We and others [13-16] have shown that Dickkopf-1 (Dkk-1), which is upregulated by GC use, suppresses the transduction of the Wnt/ $\beta$-catenin cascade in BMSCs and promotes their differentiation into adipocytes, implying a potential role of Dkk-1 in the pathogenesis of GCinduced ONFH. Wang and colleagues [17] demonstrated that Dkk-1 knockdown by an antisense oligonucleotide enhances the osteogenesis and proliferation of osteoblasts and attenuates adipocytic differentiation. Additionally, Tang et al. [18] and Hang et al. [19] enhanced the recovery of BMSCs in ONFH by introducing an exogenous BMP-2 or VEGF gene. These genes are responsible for osteoblastic lineage differentiation or angiogenesis, respectively. Based on these previous findings, we hypothesized that interference 
with the expression of Dkk-1 in BMSCs would arrest the progression of GC-induced ONFH by simultaneously suppressing the adipogenesis and enhancing the osteogenesis of BMSCs.

\section{Methods}

2.1. Isolation, Culture, and Identification of Primary BMSCs. One-week-old Sprague-Dawley rat pups were sacrificed sterilely, and both bilateral femurs and tibias were isolated cleanly. The isolated tissues were rinsed with sterile lowglucose Dulbecco's Modified Eagle Medium (DMEM), and the epiphyses and growth plates of the femurs and tibias were dissected on sterile dishes. The marrow cavities were flushed 3-4 times by inserting a needle attached to a $2 \mathrm{ml}$ syringe containing low-glucose complete medium (low-glucose DMEM with $10 \%$ fetal bovine serum), and the resultant bone marrow suspensions were harvested into $15 \mathrm{ml}$ tubes. After centrifugation at $1300 \mathrm{rpm}$ for 5 minutes, the supernatants were removed, and the cells were suspended in complete medium (low glucose) in culture flasks and incubated in a humidified chamber at $37^{\circ} \mathrm{C}$ with $5 \% \mathrm{CO}_{2}$. After $48 \mathrm{~h}$ of primary culture, nonadherent cells were removed by replacing the medium with fresh medium. The medium was replaced every 2 days thereafter. To verify the BMSCs, 3rd-generation cells were subjected to morphological observations, osteogenic and adipogenic induction, and phenotypic analyses.

2.2. Lentivirus-Meditated Dkk-1 RNAi Vector Construction and Analysis of Transfection Efficiency. The lentiviral vectors for Dkk-1 RNAi (sequence: GTACAAATCTGCCTGGCTT) and its negative control counterpart (sequence: TTCTCC GAACGTGTCACGT) were designed and constructed by GeneChem Inc. (see the flowchart in Figure 2 of the supplementary data). The transfection efficiency was optimal when viral dosage (defined by multiplicity of infection, MOI) reached to 25 . (MOI=25) (Figure 3, supplementary data).

2.3. In Vitro Lentivirus-Meditated Dkk-1 RNA Interference and Glucocorticoid Treatments. When their primary progenitors were passaged to the 3rd generation, the subcultured BMSCs were divided into 4 groups and seeded onto separate 6-well plates: the (1) transfection group (TG); (2) GC group (GG); (3) negative group (NG); and (4) blank group (BG). When the cells in each well of the 4 plates reached $30 \%$ confluence, TG and NG were transfected with the Dkk-1 RNAi vector or negative control vector, respectively. After $12 \mathrm{~h}$ and $48 \mathrm{~h}$, the medium in each plate was renewed with low-glucose complete medium. At $96 \mathrm{~h}$ after transfection, the medium in TG, GG, and NG was replaced with fresh medium containing $10 \mu \mathrm{M}$ dexamethasone (Dex), whereas in BG, the medium was renewed as before. All groups were consecutively cultured for 14 days before subsequent analysis.

2.4. Real-Time qPCR Analysis. A two-step PCR method was applied. Briefly, total RNA was isolated from the BMSCs using a Bioteke kit (Bioteke Corporation), followed by cDNA synthesis using an iScript ${ }^{\mathrm{TM}} \mathrm{cDNA}$ synthesis kit (Bio-Rad). The primers for Ctnnb1, Runx2, Dkk-1, Gsk3b, Pparg, and Gapdh (primer sequences are listed in Table 1 of the supplementary data) were synthesized by Beijing Tsingke Biological Technology. The qPCR followed the protocol for the SsoFast EvaGreen supermix kit (Bio-Rad) with the following cycling parameters in the LightCycler ${ }^{\circledR} 96$ System: predenaturation at $95^{\circ} \mathrm{C}$ for $30 \mathrm{~s} ; 40$ cycles of denaturation at $95^{\circ} \mathrm{C}$ for $5 \mathrm{~s}$ and annealing/extension at $50-58^{\circ} \mathrm{C}$ for $10 \mathrm{~s}$ ( 40 cycles); and melting curve analysis at $65-95^{\circ} \mathrm{C}, 10 \mathrm{sec} /$ step. The fold change in relative mRNA expression was calculated by the $2^{-\Delta \Delta \mathrm{Ct}}$ method.

2.5. Western Blotting Analysis. Cells were lysed in RIPA buffer with protease inhibitor cocktail on ice for $30 \mathrm{~min}$, followed by centrifugation at $4^{\circ} \mathrm{C}$ and $1200 \mathrm{rpm}$ for $30 \mathrm{~min}$. The supernatant was stored at $-80^{\circ} \mathrm{C}$. The protein concentration was determined by the BCA method, and equal amounts of protein were separated by SDS-PAGE. For Western blotting, the samples on the gel were transferred to PVDF membranes, and the membranes were washed with TBST for $5 \mathrm{~min}$, followed by blocking in 5\% BSA in TBST for $1 \mathrm{~h}$ and washing three times with TBST for $5 \mathrm{~min}$ each. The membranes were then incubated with primary antibodies (Runx2, PPAR $\gamma$-2, GSK-3 $\beta, \beta$-catenin, Dkk-1, and GAPDH, all $1: 1000$ dilution) overnight at $4^{\circ} \mathrm{C}$ and washed 3 times with TBST for $5 \mathrm{~min}$ each before incubation with the secondary antibody at room temperature for $1 \mathrm{~h}$. After washing the membranes with TBST three times for $5 \mathrm{~min}$ each, the samples on the membranes were visualized by ECL and scanned by Quantity One software (Bio-Rad).

2.6. Establishment of the GC-Induced ONFH Rat Model. A total of 85 adult SPF Sprague-Dawley rats (purchased from Chengdu Dossy Biological Technology Co. Ltd, weighting between 250 to $350 \mathrm{~g}$ ) were intramuscularly (i.m.) injected with lipopolysaccharide (LPS) once at a dose of $10 \mu \mathrm{g} / \mathrm{kg}$. Methylprednisolone (MPS) was then i.m. injected at a dose of $20 \mathrm{mg} / \mathrm{kg}$ once daily for the next three days. To ensure survival, all rats received gentamicin solution $\left(8 \times 10^{3} \mathrm{U} / \mathrm{d}\right)$ by gavage for the next seven days, combined with i.m. injection of lansoprazole $(1 \mathrm{mg} / \mathrm{d})$. Thereafter, all rats were carefully housed and maintained for the next 6 weeks (protocol and results in Figures 4 and 5, supplementary data).

2.7. Cell Implantation into Femoral Heads. After 6 weeks, the remaining 80 rats ( 4 rats died during the period of establishing GC-induced ONFH model, and one was excluded to ensure equal group sizes) were randomly allocated to 4 groups: the control group, which was treated with normal saline (control); the therapeutic group, which was treated with Dkk-1 RNAimodified BMSCs (LV-Dkk-1-RNAi-BMSCs, LDRM); the negative control group, which was treated with empty sequence-modified BMSCs (LV-ES-BMSCs, LEM); and the normal therapeutic group, which was treated with normal, unmodified BMSCs. Due to the small size of the rat femoral head, intrabone marrow injection was employed [20]. To perform the procedure, the animal was anesthetized using chloral hydrate, and the lower limb was disinfected. An incision was made in the center of the knee, and the skin was dissected bluntly layer-by-layer to open the knee cavity. A $2 \mathrm{ml}$ needle was inserted into the intercondylar, penetrating the 
marrow cavity of the femur and approaching beneath the trochanter major. Then, $0.1 \mathrm{ml}$ of BMSCs $\left(10^{7}\right.$ cells $\left./ \mathrm{ml}\right)$ or normal saline mixed with heparin was injected; the marrow cavity was sealed with sterilized bone wax, and the skin was sutured. Gentamycin was administered in-feed for the next week to prevent further infection, and the rats were observed for another 7 weeks. After sacrifice, the bilateral femoral heads of all rats (except those used for frozen sections) were dissected, fixed in 4\% paraformaldehyde, decalcified in EDTA solution for 3 weeks, washed with deionized water for $10 \mathrm{~min}$, dehydrated in a gradient of ethyl alcohol, waxed, embedded, and sliced into $3 \mu \mathrm{m}$ thick sections. The protocol is illustrated in brief in Figure 4 in the supplementary data.

2.8. In Vitro and In Vivo Histological Analyses. Cell samples were stained with Oil Red $\mathrm{O}$ or alizarin red in vitro. The culture medium was aspirated completely from BMSCs incubated in 6-well plates, and the cells were rinsed 3 times with PBS and fixed in $4 \%$ paraformaldehyde for $10 \mathrm{~min}$. After rinsing 3 times with PBS, the cells were incubated with Oil Red O solution (Sigma, USA) or alizarin red (Sigma, USA) at $37^{\circ} \mathrm{C}$ for $20 \mathrm{~min}$. Excess solution was then removed by rinsing with distilled water, followed by staining with hematoxylin for $2 \mathrm{~min}$. After rinsing again with distilled water, the cells were mounted in aqueous mounting medium and observed by phase contrast microscopy.

Frozen sections of the femoral heads of rats were also subjected to Oil Red $\mathrm{O}$ staining. The frozen slices were prepared and placed in absolute propylene glycol for $5 \mathrm{~min}$. The slices were then stained with Oil Red O solution (Sigma, USA) for $20 \mathrm{~min}$, followed by differentiation in $85 \%$ propylene glycol for $2 \mathrm{~min}$. After rinsing 3 times with distilled water, the slices were stained with hematoxylin for $1 \mathrm{~min}$, rinsed with distilled water, and mounted on a coverslip in aqueous mounting medium. All slices were observed by microscopy.

Hematoxylin and eosin (H\&E) staining was performed on paraffin slices of the femoral heads of rats as follows: the slices were dewaxed in xylene, dehydrated in a gradient of ethyl alcohol, rinsed 3 times with distilled water, stained with hematoxylin, differentiated in $1 \% \mathrm{HCl}$ ethanol for $30 \mathrm{~s}$, washed with running tap water for $15 \mathrm{~min}$, stained with eosin, washed with running tap water for $3 \mathrm{~min}$, dehydrated again in a gradient of ethyl alcohol, vitrified in xylene, and finally mounted in resinene.

2.9. Immunohistochemical Analysis. Femoral head slices were dewaxed and hydrated, followed by antigen retrieval in sodium citrate ( $\mathrm{pH} 6.0$ ) at $95^{\circ} \mathrm{C}$ for $40 \mathrm{~min}$. After cooling to room temperature, peroxidases were inactivated by incubating the slices in $3 \% \mathrm{H}_{2} \mathrm{O}_{2}$ for $15 \mathrm{~min}$. The slices were incubated in a humid atmosphere with diluted primary antibodies (Dkk-1, GSK-3 $\beta, \beta$-catenin, PPAR $\gamma 2$, and Runx2) at $37^{\circ} \mathrm{C}$ for $45 \mathrm{~min}$, washed 3 times with PBS, and then incubated with biotin-conjugated secondary antibody at $37^{\circ} \mathrm{C}$ for $45 \mathrm{~min}$. HRP and $100 \mu \mathrm{l}$ of DAB was then added. Staining was monitored under a microscope, and the reactions were terminated by the addition of distilled water. The slices were then stained with hematoxylin, dehydrated in a gradient of ethyl alcohol, vitrified in xylene, mounted in neutral resin, and evaluated by light microscopy in a 400-fold magnified field. Five fields were randomly selected and captured by Image-Pro Plus 6.0 to calculate the percentage of areas of positive expression.

2.10. Statistical Analyses. All statistical analyses were performed using SPSS 17.0 software, and differences were considered significant at $P<0.05$. ANOVA with multiple comparisons was used to analyze all parametric data. The incidence of GC-induced ONFH in rats was assessed by $\chi^{2}$ tests with multiple comparisons. All bar plots were prepared in ggplot2 (version 2.21).

\section{Results}

3.1. The Effects of GC Treatment and Lentivirus-Meditated Dkk-1 RNAi on the Osteogenesis and Adipogenesis of BMSCs In Vitro. After consecutive 14-day cultivation of all BMSC groups, in vitro adipogenesis or osteogenesis was visualized by Oil Red $\mathrm{O}$ or alizarin red $\mathrm{S}$ staining, respectively. As shown in Figures 1(a)-1(d), larger areas of red staining indicated greater lipid accumulation and adipogenesis. Adipogenic differentiation was much lower in BG and TG than in GG and NG. Consistent with these results, more calcium formation was observed in BG and TG than in GG and NG, as demonstrated by the area of red staining (Figures 1(e)$1(\mathrm{~h})$ ), indicating greater osteogenesis in both BG and TG than in GG and NG.

3.2. In Vitro RT-PCR and Western Blotting Analyses of Dkk-1, GSK-3 $\beta$, $\beta$-Catenin, Runx 2 , and PPAR $\gamma$. The RNA and protein expression of GSK-3 $\beta$ and $\beta$-catenin, members of the canonical Wnt pathway, and Dkk-1, the extracellular antagonist of this cascade, were assessed by RT-PCR and Western blot, respectively. The expression of GSK-3 $\beta$ and $\beta$-catenin was significantly lower and higher, respectively, in BG and TG than in the other two groups (Figures 2(B) and 2(G); Figures $2(\mathrm{C})$ and Figure $2(\mathrm{H}) ; P<0.05)$. The expression of Dkk-1 (Figures 2(A) and 2(F)) was also significantly lower in BG and TG $(P<0.05)$ than in the other two groups. By contrast, Runx2 and PPAR $\gamma$ (Figures 2(D) and 2(I); Figures 2(E) and 2(J)), globally accepted markers of osteogenesis or adipogenesis, respectively, exhibited opposing patterns of expression. Runx2 expression was significantly higher in BG and TG than in GG and NG $(P<0.05)$, whereas PPAR $\gamma$ expression was significantly lower in BG and TG than in GG and NG $(P<0.05)$ (Figure 2).

3.3. In Vivo HeE Staining of the Femoral Heads of Rats. Eight weeks after the operation, all 80 rats were sacrificed, and $\mathrm{H} \& \mathrm{E}$ staining was performed. The diagnostic criterion of ONFH was empty lacunae. Briefly, ten fields (20x) from an $\mathrm{H} \& \mathrm{E}$ staining section of one rat were randomly selected to count empty lacunae. If empty lacunae were found in over 5 fields (empty lacunae percentage $>50 \%$ ), ONFH was diagnosed in a rat. In the control group, most of the trabeculae were cracked, discontinuous, and reduced, and the mean percentage of empty lacunae was $67.7 \% \pm 13.8 \%$, confirming the presence of ONFH. These histological phenomena were observed in 18 of 20 rats, corresponding to an incidence of ONFH of 


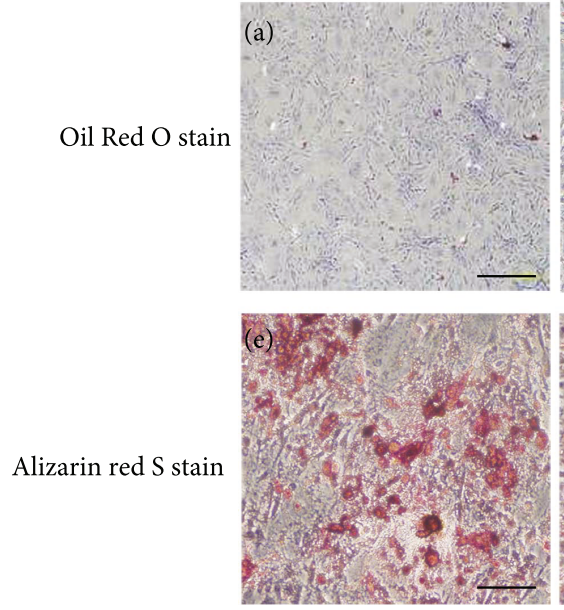

BG


GG
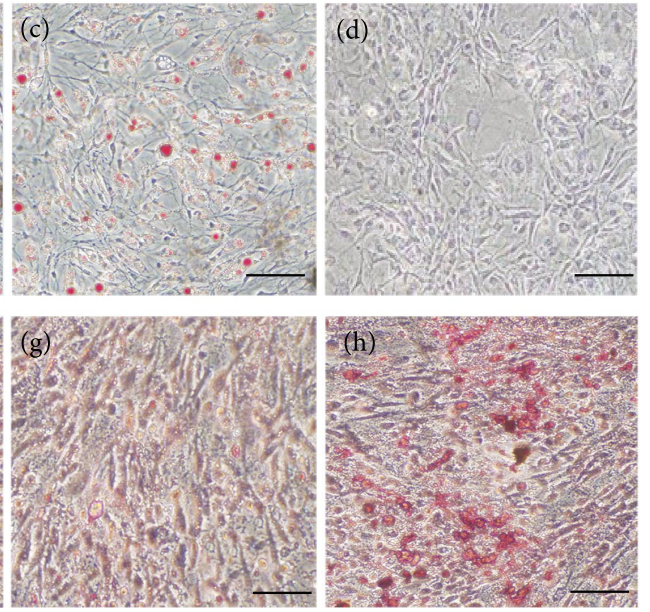

NG

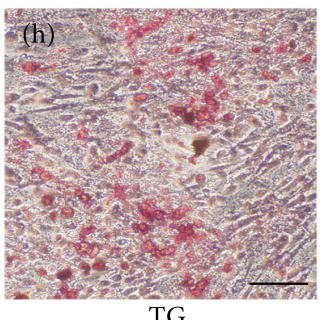

TG

FIGURE 1: Oil Red O staining indicating the adipogenesis (red droplets for lipid, $(\mathrm{a}-\mathrm{d})$ ) and alizarin red staining indicating osteogenesis (red nodules for mineralized calcium, (e-h)). No sign of red droplets in the blank group (BG, (a)) or the transfection group (TG, (d)) meant no lipid formation in the two groups. While in GC group (GG, (b)) and negative group (NG, (c)), significant red droplets suggested more lipid formation. On the other hand, BG and TG formed significantly more mineralized calcium than the GC and NG.

$90 \%$ in control group. In the LDRM group, normal, continuous trabeculae were observed in 13 rats, with an empty lacunae percentage far below $50 \%$; ONFH was identified in only 7 rats, corresponding to an overall incidence of $35 \%$. The mean percentage of empty lacunae in all 20 rats was $31.8 \% \pm 19.0 \%$. By comparison, 55\% (11/20) of the rats in the LEM group developed $\mathrm{ONFH}$, with a mean empty lacuna percentage of $48.7 \% \pm 14.6 \%$; in the BMSC group, the incidence of ONFH was $50 \%(10 / 20)$, and the mean percentage of empty lacunae was $50.4 \% \pm 18.1 \%$. The differences in ONFH incidence and the mean percentage of empty lacunae between the control group and the other groups were statistically significant $(P<0.05)$. However, the differences among the other three groups were not significant $(P>0.05)$ (Figure 3 and Table 1 ).

\subsection{In Vivo Oil Red O Staining of the Femoral Head. Eight} weeks after the operation, Oil Red $\mathrm{O}$ staining was performed to visualize adipocytes in femoral head samples from the 4 groups. Red-stained cells in each rat specimen were counted under three different 40x fields. The number of red-stained cells was highest in the control group, with a mean of 17.05 \pm 7.03 , followed by $6.21 \pm 3.06,10.32 \pm 4.12$, and $9.97 \pm 4.35$ in the LDRM, LEM, and BMSC groups, respectively. Only the differences between the control group and the other three groups were significant $(P<0.05)$ (Figure 4$)$.

3.5. In Vivo Immunohistochemical Analyses of the Expression of Dkk-1, PPAR $\gamma, G S K-3 \beta, R u n x 2$, and $\beta$-Catenin in the Femoral Head. The expression of five proteins, Dkk-1, $\operatorname{PPAR} \gamma, \mathrm{GSK}-3 \beta$, Runx 2 , and $\beta$-catenin, in the rat femoral head, was immunohistochemically analyzed, and the number of positive cells was quantified by Image-Pro Plus (ver. 6.0). The percentage of Dkk-1-positive cells was significantly $(P<0.05)$ higher in the control group (mean positive rate of $65.41 \% \pm 14.07 \%$ ) than in the other groups (LDRM, $20.35 \% \pm 8.80 \%$; LEM, $38.98 \% \pm 11.69 \%$; BMSC, $44.66 \% \pm$ $7.83 \%)$. In addition, the Dkk-1-positive staining intensity was significantly lower in LDRM than in either LDRM or BMSC $(P<0.05)$. Similarly, the percentage of PPAR $\gamma$ or GSK-3 $\beta$-positive cells was significantly $(P<0.05)$ higher in the control group $(43.14 \% \pm 10.85 \%$ and $34.54 \% \pm 7.77 \%$, resp.) than in the other three groups (LDRM, $16.77 \% \pm$ $6.93 \%$ and $10.08 \% \pm 8.14 \%$; LEM, $27.08 \% \pm 9.43 \%$ and $17.70 \% \pm 9.66 \%$; BMSC, $26.45 \% \pm 7.22 \%$ and $16.68 \% \pm$ $5.45 \%)$. By contrast, the percentages of positive staining for Runx 2 and $\beta$-catenin were significantly $(P<0.05)$ lower in the control group $(8.78 \% \pm 4.30 \%$ and $4.21 \% \pm 1.45 \%$, resp.) than in the other three groups. Positive staining for Runx2 and $\beta$-catenin was highest in LDRM $(32.50 \% \pm 7.12 \%$ and $13.13 \% \pm 2.77 \%)$, but these values did not differ significantly $(P>0.05)$ from those in LEM $(23.74 \% \pm 8.45 \%$ and $9.05 \% \pm 2.64 \%)$ or BMSC $(25.01 \% \pm 6.67 \%$ and $10.51 \% \pm$ $1.70 \%$ ) (Figure 5).

\section{Discussion}

Although clinical success in BMSC implantation has been reported, the treated ONFH cases were heterogeneous in etiology [3, 6, 21]: trauma, glucocorticoid use, alcohol abuse, and idiopathic. This etiological heterogeneity may reduce the validity of these results due to low osteogenesis or proliferation of autologous BMSCs in patients with GC-induced ONFH [10-12]. Hence, we hypothesized that implanting BMSCs from healthy individuals in those with GC-induced $\mathrm{ONFH}$ might produce superior outcomes compared to implantation of autologous cells.

Moreover, there are new therapeutic strategies for ONFH involving modifications of genes encoding stem cellgenerating growth factors, such as bone morphogenetic protein-2 (BMP-2) and vascular endothelial growth factor (VEGF) [22-24]. Tang and colleagues [18] repaired surgically induced $\mathrm{ONFH}$ in goats by introducing exogenous BMP-2 in BMSCs. In another study, Hang [19] and colleagues successfully induced bone regeneration in $\mathrm{ONFH}$ 


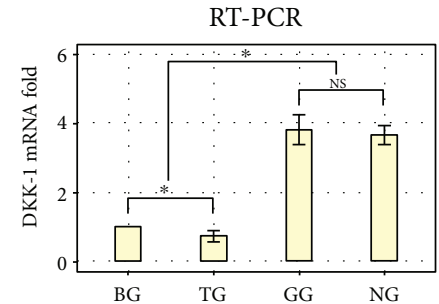

(A)

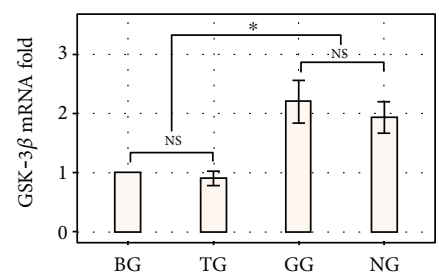

(B)

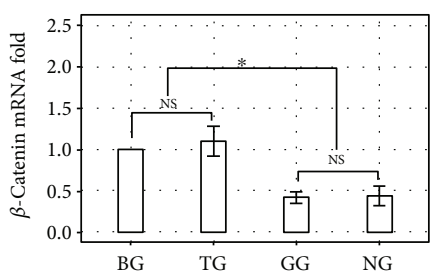

(C)



(D)

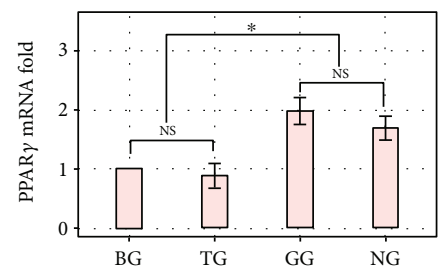

(E)

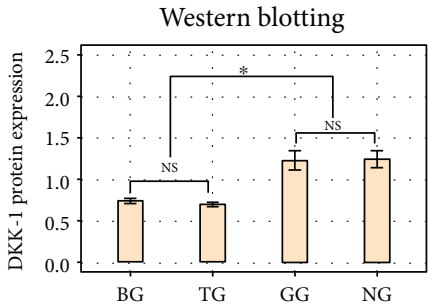

(F)

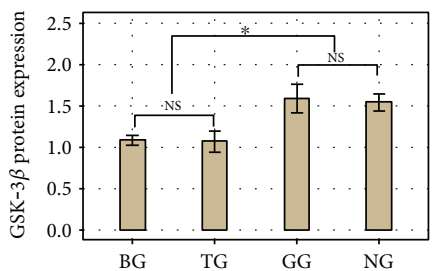

(G)

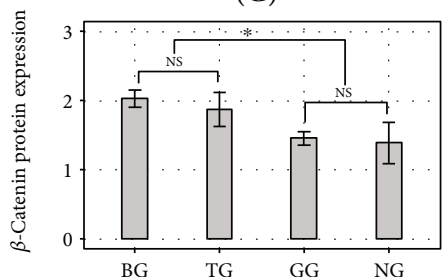

(H)

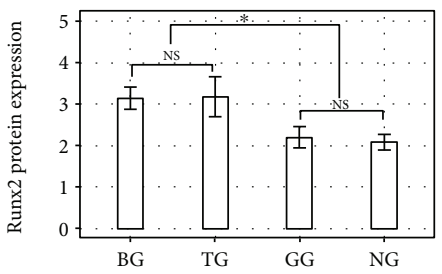

(I)

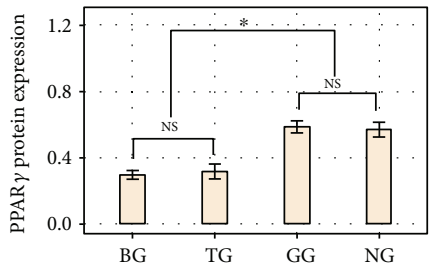

(J)

(b)

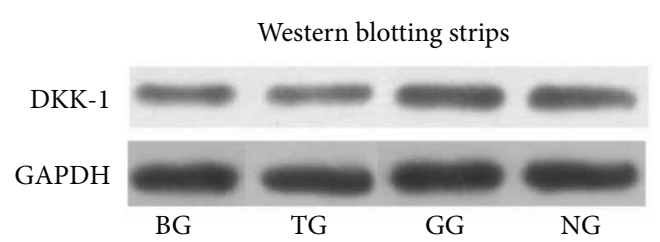

GSK-3 $\beta$
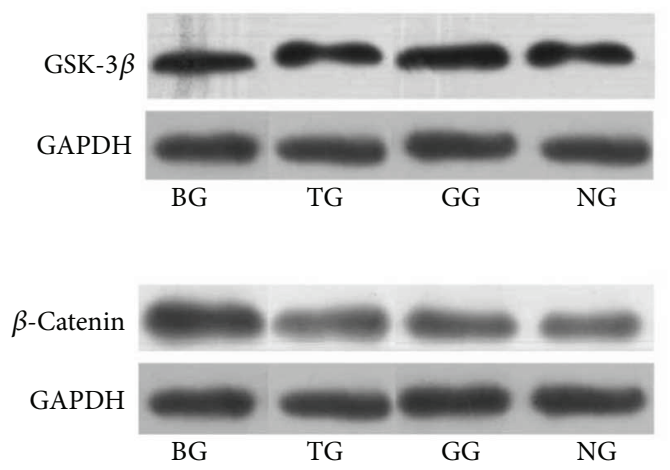

Runx2

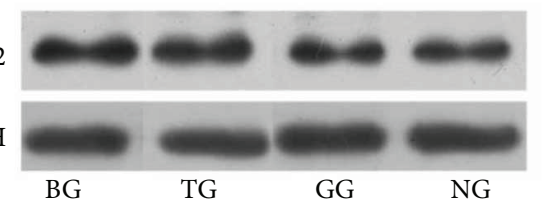

GAPDH

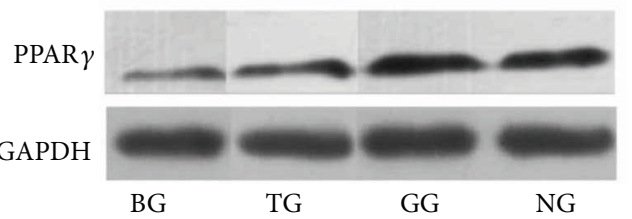

(c)

FIGURE 2: RT-PCR analyses (a) for mRNA levels of Dkk-1, GSK3 $\beta$, $\beta$-catenin, Runx2, and PPAR $\gamma$. Dkk-1, the antagnosist of Wnt/ $\beta$-catenin pathway, its mRNA level in TG were significantly lower than BG, GG or NG $(P<0.05)$ but not between GG and NG $(P>0.05)$. GSK3 $\beta$ mRNA in BG or TG was significantly lower $(P<0.05)$ than that of GG or NG, and mRNA of $\beta$-catenin in TG or BG was higher $(P<0.05)$ than that in GG or NG. Runx2 was higher $(P<0.05)$ in BG or TG than that in GG or NG while PPAR $\gamma$ was lower $(P<0.05)$ in BG or TG than that in GG or NG. The mRNA level of GSK3 $\beta, \beta$-catenin, Runx2, or PPAR $\gamma$ was not significantly different between BG and TG $(P>0.05)$ or between GG and NG $(P>0.05)$. Western blotting analyses (b and $\mathrm{c})$ of the expressions of Dkk-1, GSK3, $\beta$-catenin, Runx2, and PPAR $\gamma$. The amount of Dkk-1 or GSK3 $\beta$ within TG and BG had no difference $(P>0.05)$, as well as within GG and NG $(P>0.05)$. But in TG or BG, these amounts were lower than those in GG or NG $(P<0.05)$. So, $\beta$-catenin, as the intracellular signal transducer of $\mathrm{Wnt} / \beta$-catenin pathway, its expression were significantly higher in BG or TG (no difference between these two groups, $P>0.05$ ) than those in GG or NG (no difference between these two groups, $P>0.05$ ). The expression of osteogenesis marker, Runx2, was also more upregulated $(P<0.05)$ in BG or TG than that in GG or NG. On the contrary, the adipogenesis marker, PPAR $\gamma$, had the opposite expression: lower in BG or TG and higher in GG or NG. Also, the expressions of Dkk-1, GSK3 $\beta, \beta$-catenin, Runx2, and PPAR $\gamma$ had no statistical significances $(P>0.05)$ between $\mathrm{BG}$ and TG or between GG and NG $\left(\mathrm{NS}, P>0.05 ;{ }^{*} P<0.05\right)$.

animals by implanting BMSCs transfected with VEGF. Our previous studies [13-16] suggested that GC-induced extracellular Dkk-1 upregulation and the resultant interruption of intracellular $\mathrm{Wnt} / \beta$-catenin transduction not only reduce the differentiation and lifespan of osteoblasts or osteocytes but also alter the osteogenesis of BMSCs toward 


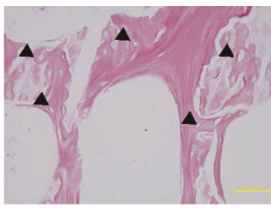

Control

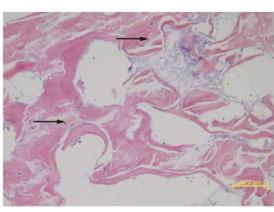

LEM

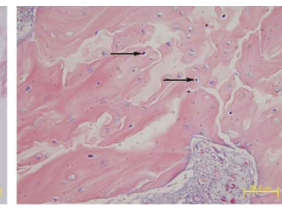

LDRM

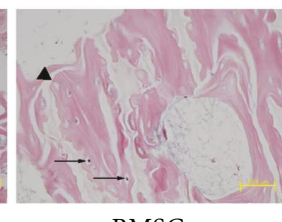

BMSC (a)

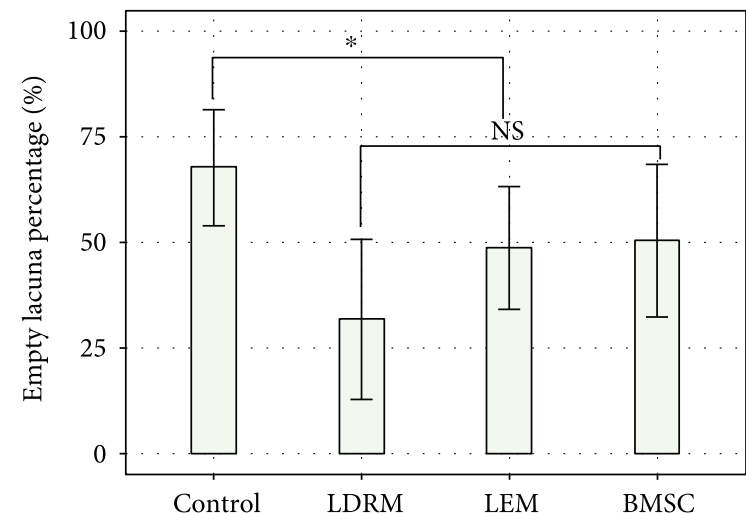

(b)

FIGURE 3: H\&E staining of femoral head specimens from 4 groups of rats (a) and the statistical data for the empty lacuna rate (b). Thinner trabecula and higher mean empty lacuna (black triangle) percentage in the control group $(67.7 \% \pm 13.8 \%, P<0.05)$ than that in others. Normal trabecula, more living osteocytes (black arrow), and lower empty lacuna percentage $(31.8 \% \pm 19.0 \%$ in LDRM, $48.7 \% \pm 14.6 \%$ in LEM, and $50.4 \% \pm 18.1 \%$ in BMSC; $P>0.05$ ) in the rest of the three groups (scale bar $=50 \mu \mathrm{m} . \mathrm{NS}, P>0.05 ;{ }^{*} P<0.05$ ).

TABLE 1: Incidence of GC-induced ONFH in each group.

\begin{tabular}{lccc}
\hline Group & Yes & GC-induced ONFH & \\
& Control** & No & Total \\
LDRM $^{*}$ & 18 & 2 & 20 \\
LEM $^{*}$ & 7 & 9 & 20 \\
BMSC $^{*}$ & 11 & 10 & 20 \\
\hline
\end{tabular}

${ }^{*} P>0.05$ for comparisons among LDRM, LEM, and BMSC. ${ }^{* *} P<0.05$ compared to the other three groups.

adipogenesis. Our findings and those of others [17, 25, 26] rationally support a potential role of these abnormalities in the pathogenesis of GC-induced ONFH. Consistent with this role, inhibition of Dkk-1 shifts adipogenesis [14] toward osteogenesis and promotes bone formation [27]. Thus, it is reasonable to propose the implantation of Dkk-1 RNAimodified BMSCs to simultaneously prevent the onset of $\mathrm{GC}$-induced ONFH and reverse its progress.

In vitro, glucocorticoid (GC) treatment induced the upregulation of Dkk-1 at both the mRNA and protein levels. This increase in Dkk-1 expression resulted in activation of GSK-3 $\beta$, a downstream inhibitory factor for $\beta$-catenin, and in turn, downregulation of $\beta$-catenin. GSK- $3 \beta$ and $\beta$-catenin are members of the canonical Wnt signaling pathway, consistent with previous suggestions of suppression of this pathway via enhancement of Dkk-1 by GC treatment as a possible mechanism of onset of GC-induced ONFH. Reduction of Dkk-1 expression by RNAi blocked its subsequent anti$\mathrm{Wnt} / \beta$-catenin effects induced by GC and shifted the differentiation of BMSCs from adipocytes toward osteoblasts.

In vivo, $\mathrm{H} \& \mathrm{E}$, Oil Red $\mathrm{O}$, and immunohistochemical staining revealed less GC-induced ONFH development in the treated groups (LDRM, LEM, and BMSC), as evidenced by fewer empty lacunae, a lower percentage of bone necrosis, and less adipogenesis compared with the untreated group (control). However, the differences between the three treated groups, LDRM and LEM, were not statistically significant, in contrast to the significant differences in mRNA and protein expression and Oil Red $\mathrm{O}$ staining between TG and NG in vitro. Also, the in vivo results revealed that LDRM group was not significantly improved, compared with the BMSC group whose GC-induced rats treated with normal and unmodified BMSCs. Thus, although BMSCs modified by lentivirus-meditated Dkk-1 RNAi were able to activate the $\mathrm{Wnt} / \beta$-catenin pathway, inhibit continuous GC-induced adipogenesis, and maintain osteogenesis in vitro, their ability to arrest the progress of GC-induced ONFH in vivo was not significantly superior to that of negative control-transfected or even unmodified BMSCs (BMSC group).

The discrepancies in the results of the in vitro and in vivo studies may be attributed to differences in protocol. In vitro, we first reduced Dkk-1 expression in BMSCs by introducing lentivirus-meditated RNAi and then subsequently exposed the cells to GC to assess the protective effects of this modification. Performing transfection prior to GC exposure is a common sequence in studies of the effects of gene modifications in the pathogenesis of GC-induced ONFH [28]. For example, Yun and coworkers [29] firstly silence the expression of GSK-3 $\beta$ in osteoblasts by siRNA transfection, and then the cells were treated with Dex to assess that whether osteoblasts with GSK-3 $\beta$ silence could resist to the Dexinduced apoptosis. Likewise, Butler et al. [30] also knocked down the expression of Dkk-1 in osteoblasts in the first place and then exposed the cells to Dex.

However, the protocols of studies of stem cell therapies for GC-induced ONFH differ from those of pathogenesis research. In general, GC-induced ONFH animal models are established $[31,32]$ or patients are recruited, both prior to the stem cell treatments, with no subsequent GC exposure in the remainder of the study. Wen and workmates [32] established the GC-induced ONFH rabbits with a combination of LPS $(10 \mu \mathrm{g} / \mathrm{kg}$ body weight, one injection) and MPS $(20 \mathrm{mg} / \mathrm{kg}$ body weight, three times injection) injection in the first place and then transplanted BMSCs which were hepatocyte growth factor (HGF)-overexpressed 


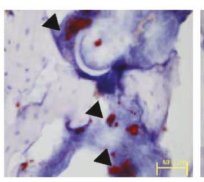

Control

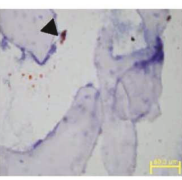

LDRM

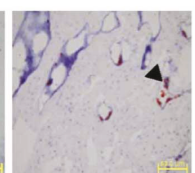

LEM

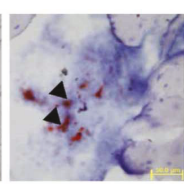

BMSC

(a)

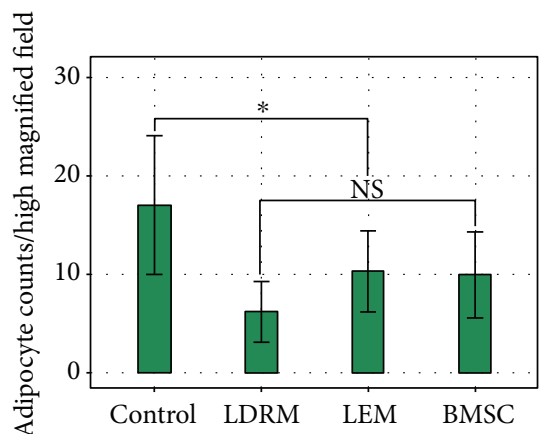

(b)

FIGURE 4: In vivo Oil Red O staining of femoral heads from rats (a) and the measured data (b). The control group contained significantly more adipocytes (black triangle) per high magnified filed $(17.05 \pm 7.03, P<0.05)$ than the other groups $(6.21 \pm 3.06,10.32 \pm 4.12$, and $9.97 \pm 4.35$ in LDRM, LEM, and BMSC, resp., $P>0.05)$, and the cell size was also larger in the control group than that in others $($ scale bar $=50 \mu \mathrm{m}$. NS, $\left.P>0.05 ;{ }^{*} P<0.05\right)$.
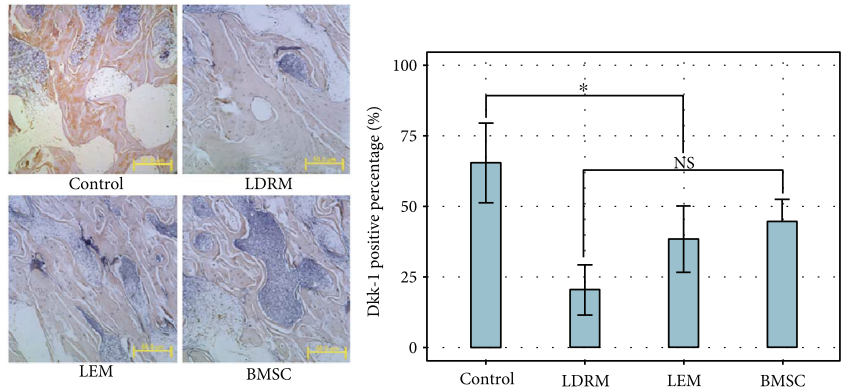

(a)
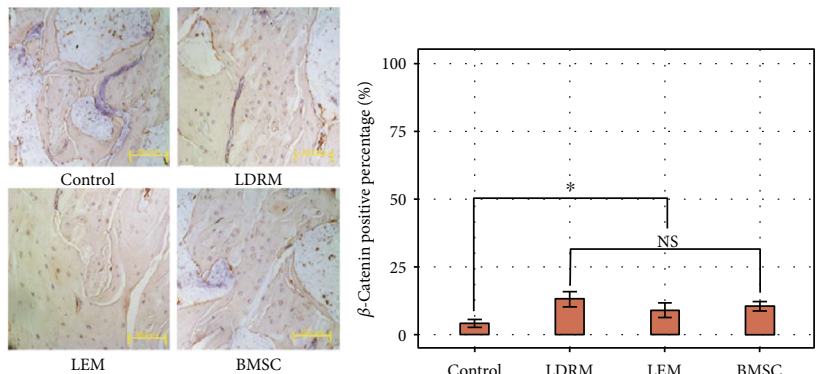

(c)
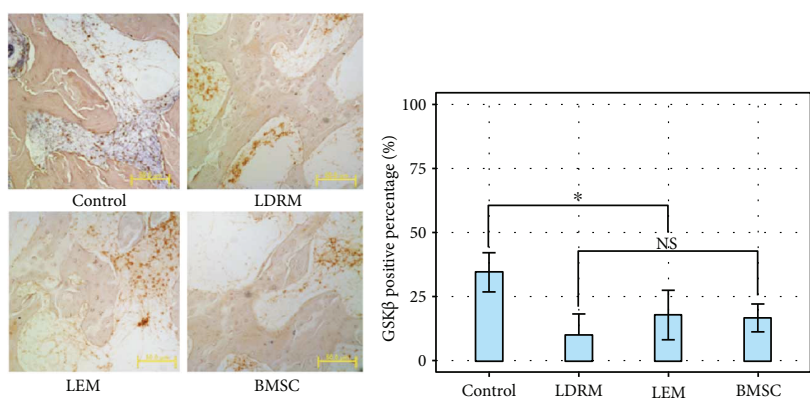

(b)
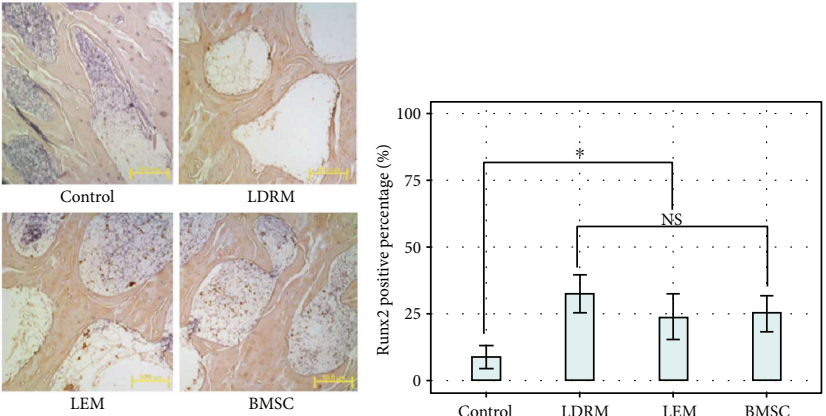

(d)
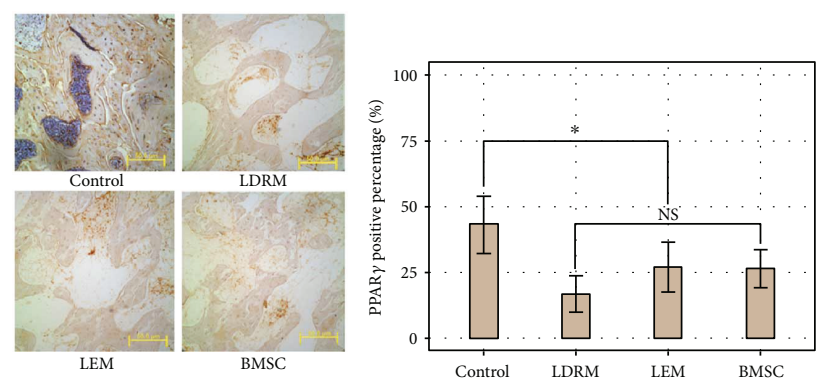

(e)

FIGURE 5: Immunohistochemical analyses for expression of Dkk-1(a), GSK3 $\beta$ (b), $\beta$-catenin (c), Runx2 (d), or PPAR $\gamma$ (e) in femoral head specimens from 4 group rats. Dkk-1 positive percentage in the control group was stronger than the rest, while the positive rate in LDRM was significantly weaker than LEM or BMSC. GSK-3 $\beta$ positive. Similarly, GSK3 $\beta$ positive rate in the control group was significantly stronger than others $(P<0.05)$. Positive rate of $\beta$-catenin or Runx 2 was significantly higher in LDRM, LEM, or BMSC (but $P>0.05$ within these three) than the control group $(P<0.05)$. PPAR $\gamma$, with its strongest positive rate in the control group, was significantly higher than the LDRM, LEM, or BMSC $\left(P>0.05\right.$ within these three as well) (scale bar $=50 \mu \mathrm{m}$. NS, $\left.P>0.05 ;{ }^{*} P<0.05\right)$. 
to treat this disease. Ding et al. [33] also used the similar treated process: inducing ONFH animal model firstly and then the Hif- $1 \alpha$ transgenic BMSCs were transplanted to the necrotic site.

In vitro, GC-induced Dkk-1 activation clearly impaired the $\mathrm{Wnt} / \beta$-catenin pathway, with an increase in adipogenesis and decrease in osteogenesis; only the group in which Dkk-1 was downregulated, BMSC, escaped this fate. However, although studies have demonstrated that deletion of Dkk-1 significantly increases $\mathrm{Wnt} / \beta$-catenin expression and bone formation in vivo [27], a corresponding increase in $\mathrm{Wnt} / \beta$ catenin expression was not observed in the group treated with BMSCs in which Dkk-1 was downregulated (LDRM) compared with the groups that received BMSCs without genetic modification (LEM and BMSC). This discrepancy with the previous report [27] indicates further study. A gene therapy pattern reported by Zhang et al. [31] could be helpful. After establishing GC-induced ONFH rabbit model, Zhang and colleagues directly injected adeno-associated virus vector which carried both VEGF and BMP genes into the femoral head via core decompression tunnel.

The present study is subject to limitations that may reduce the validity of our conclusions. First, the bone mass of the femoral head, an indicator of new bone formation, was not quantified in each treated group. Second, the implanted cells were allogeneic BMSCs. Although transplantation of allogeneic BMSCs has been used previously to treat patients with ONFH [34] or other joint diseases [35-37], the immunological safety of these allogeneic BMSCs remains unclear [38-40]. Further studies are necessary.

\section{Conclusion}

BMSCs with lentivirus-meditated Dkk-1 RNAi not only prevented the GC-induced decrease in the $\mathrm{Wnt} / \beta$-catenin cascade, normalized the expression of the osteogenic marker Runx2, and attenuated the adipogenic marker PPAR $\gamma$ but also maintained osteogenesis rather than adipogenesis under GC exposure. Moreover, the implantation of these Dkk-1downregulated BMSCs into the necrotic femoral head in rats with GC-induced ONFH prevented progression of the disease, although the effects were not significantly superior to treatment with nongenetically modified BMSCs.

\section{Conflicts of Interest}

The authors have no conflicts of interest to declare.

\section{Acknowledgments}

This work was funded by the National Natural Science Foundation of China (Grant no. 81171763).

\section{Supplementary Materials}

Figure 1: BMSC morphological observation (a), phenotypic analyses (b), and osteogenetic and adipogenic induction (c and $d$ ). Figure 2: full workflow of constructing the lentivirus-meditated vector and negative controlled counterpart. Figure 3: Transfection effect and optimal MOI analyses. From A to E, MOI: 0, 15, 25, 35, and 45. Figure 4: the protocol of GC-induced ONFH establishment and the cell implantations. Figure 5: H\&E staining of femoral heads in GC-induced ONFH rats (a: GC group; b: normal controlled group). GC group with thinner trabecula and significantly more empty lacuna. Scale bar $=50.0 \mu \mathrm{m}$. (Supplementary Materials)

\section{References}

[1] Y. Bian, W. Qian, H. Li, R. C. Zhao, W. X. Shan, and X. Weng, "Pathogenesis of glucocorticoid-induced avascular necrosis: a microarray analysis of gene expression in vitro," International Journal of Molecular Medicine, vol. 36, no. 3, pp. 678-684, 2015.

[2] Y.-L. Zhang, J.-H. Yin, H. Ding, W. Zhang, C.-Q. Zhang, and Y.-S. Gao, "Vitamin $\mathrm{K}_{2}$ prevents glucocorticoid-induced osteonecrosis of the femoral head in rats," International Journal of Biological Sciences, vol. 12, no. 4, pp. 347-358, 2016.

[3] C. Papakostidis, T. H. Tosounidis, E. Jones, and P. V. Giannoudis, "The role of "cell therapy" in osteonecrosis of the femoral head. A systematic review of the literature and meta-analysis of 7 studies," Acta Orthopaedica, vol. 87, no. 1, pp. 72-78, 2016.

[4] N. S. Piuzzi, J. Chahla, H. Jiandong et al., "Analysis of cell therapies used in clinical trials for the treatment of osteonecrosis of the femoral head: a systematic review of the literature," The Journal of Arthroplasty, vol. 32, no. 8, pp. 2612-2618, 2017.

[5] V. Gangji, V. De Maertelaer, and J.-P. Hauzeur, “Autologous bone marrow cell implantation in the treatment of nontraumatic osteonecrosis of the femoral head: five year followup of a prospective controlled study," Bone, vol. 49, no. 5, pp. 1005-1009, 2011.

[6] N. S. Piuzzi, J. Chahla, J. B. Schrock et al., "Evidence for the use of cell-based therapy for the treatment of osteonecrosis of the femoral head: a systematic review of the literature," The Journal of Arthroplasty, vol. 32, no. 5, pp. 1698-1708, 2017.

[7] T. Asada, T. Kushida, M. Umeda et al., "Prevention of corticosteroid-induced osteonecrosis in rabbits by intra-bone marrow injection of autologous bone marrow cells," Rheumatology, vol. 47, no. 5, pp. 591-596, 2008.

[8] R. K. Sen, S. K. Tripathy, S. Aggarwal, N. Marwaha, R. R. Sharma, and N. Khandelwal, "Early results of core decompression and autologous bone marrow mononuclear cells instillation in femoral head osteonecrosis: a randomized control study," The Journal of Arthroplasty, vol. 27, no. 5, pp. 679686, 2012

[9] D. Zhao, D. Cui, B. Wang et al., "Treatment of early stage osteonecrosis of the femoral head with autologous implantation of bone marrow-derived and cultured mesenchymal stem cells," Bone, vol. 50, no. 1, pp. 325-330, 2012.

[10] Y. W. Lim, Y. S. Kim, J. W. Lee, and S. Y. Kwon, "Stem cell implantation for osteonecrosis of the femoral head," Experimental \& Molecular Medicine, vol. 45, no. 11, article e61, 2013.

[11] M. T. Houdek, C. C. Wyles, B. D. Packard, A. Terzic, A. Behfar, and R. J. Sierra, "Decreased osteogenic activity of mesenchymal stem cells in patients with corticosteroid-induced osteonecrosis of the femoral head," The Journal of Arthroplasty, vol. 31, no. 4, pp. 893-898, 2016.

[12] B.-L. Wang, W. Sun, Z.-C. Shi et al., "Decreased proliferation of mesenchymal stem cells in corticosteroid-induced 
osteonecrosis of femoral head," Orthopedics, vol. 31, no. 5, p. 444, 2008.

[13] G. Tan, P. D. Kang, F. X. Pei et al., "Comparing study on expression levels of related factors in glucocorticoid-induced osteonecrosis of femoral head and hip osteoarthritis," Chinese Journal of Joint Surgery, vol. 6, no. 2, pp. 59-62, 2012.

[14] X. Xie, P. Kang, F. Pei et al., "DKK-1 antibody can promote mouse's adipocytes to transdifferentiate into osteoblasts," Orthopedic Journal of China, vol. 21, no. 7, pp. 695-700, 2013.

[15] X. Xie, F. Pei, P. Kang, J. Yang, B. Shen, and Z. Zhou, "Effect of different concentrations, multi-treatment course of dexamethasone on osteoblast activity and Wnt signal pathway in rats," Chinese Journal of Osteoporosis, vol. 19, no. 8, pp. 823-828, 2013.

[16] L. Deng, Z. Tan, X. Xie, and P. Kang, "Application and prospects of specific inhibition of DKK-1 in the treatment of glucocorticoids-induced osteoneerosis of the femoral head," Orthopedic Journal of China, vol. 22, no. 17, pp. 1603-1606, 2014.

[17] F.-S. Wang, J.-Y. Ko, D.-W. Yeh, H.-C. Ke, and H.-L. Wu, "Modulation of Dickkopf-1 attenuates glucocorticoid induction of osteoblast apoptosis, adipocytic differentiation, and bone mass loss," Endocrinology, vol. 149, no. 4, pp. 17931801, 2008.

[18] T. T. Tang, B. Lu, B. Yue et al., "Treatment of osteonecrosis of the femoral head with hBMP-2-gene-modified tissueengineered bone in goats," The Journal of Bone and Joint Surgery, vol. 89-B, no. 1, pp. 127-129, 2007.

[19] D. Hang, Q. Wang, C. Guo, Z. Chen, and Z. Yan, "Treatment of osteonecrosis of the femoral head with $\mathrm{VEGF}_{165}$ transgenic bone marrow mesenchymal stem cells in mongrel dogs," Cells Tissues Organs, vol. 195, no. 6, pp. 495-506, 2012.

[20] N. d. M. Ocarino, J. N. Boeloni, V. Jorgetti, D. A. Gomes, A. M. Goes, and R. Serakides, "Intra-bone marrow injection of mesenchymal stem cells improves the femur bone mass of osteoporotic female rats," Connective Tissue Research, vol. 51, no. 6, pp. 426-433, 2010.

[21] H. Cao, H. Guan, Y. Lai, L. Qin, and X. Wang, "Review of various treatment options and potential therapies for osteonecrosis of the femoral head," Journal of Orthopaedic Translation, vol. 4, pp. 57-70, 2016.

[22] M. T. Houdek, C. C. Wyles, and R. J. Sierra, "Osteonecrosis of the femoral head: treatment with ancillary growth factors," Current Reviews in Musculoskeletal Medicine, vol. 8, no. 3, pp. 233-239, 2015.

[23] L. Rackwitz, L. Eden, S. Reppenhagen et al., "Stem cell- and growth factor-based regenerative therapies for avascular necrosis of the femoral head," Stem Cell Research \& Therapy, vol. 3, no. 1, p. 7, 2012.

[24] Q. Wen, L. Ma, Y.-P. Chen, L. Yang, W. Luo, and X.-N. Wang, "Treatment of avascular necrosis of the femoral head by hepatocyte growth factor-transgenic bone marrow stromal stem cells," Gene Therapy, vol. 15, no. 23, pp. 1523-1535, 2008.

[25] R. S. Weinstein, "Glucocorticoid-induced osteoporosis and osteonecrosis," Endocrinology and Metabolism Clinics of North America, vol. 41, no. 3, pp. 595-611, 2012.

[26] J. Li, N. Zhang, X. Huang et al., "Dexamethasone shifts bone marrow stromal cells from osteoblasts to adipocytes by C/ EBPalpha promoter methylation," Cell Death \& Disease, vol. 4, no. 10, article e832, 2013.
[27] F. Morvan, K. Boulukos, P. Clément-Lacroix et al., "Deletion of a single allele of the $D k k 1$ gene leads to an increase in bone formation and bone mass," Journal of Bone and Mineral Research, vol. 21, no. 6, pp. 934-945, 2006.

[28] H. Dong-Hua, L. Xia, C. Lei et al., "RIPK1 axis inhibits glucocorticoid induced-apoptosis of mesenchymal stem cells in vitro," Fudan University Journal of Medical Science, vol. 42, no. 3, pp. 307-312, 2015.

[29] S.-I. Yun, H.-Y. Yoon, S.-Y. Jeong, and Y.-S. Chung, "Glucocorticoid induces apoptosis of osteoblast cells through the activation of glycogen synthase kinase $3 \beta$," Journal of Bone and Mineral Metabolism, vol. 27, no. 2, pp. 140-148, 2009.

[30] J. S. Butler, J. M. Queally, B. M. Devitt, D. W. Murray, P. P. Doran, and J. M. O'Byrne, "Silencing Dkk1 expression rescues dexamethasone-induced suppression of primary human osteoblast differentiation," BMC Musculoskeletal Disorders, vol. 11, no. 1, p. 210, 2010.

[31] C. Zhang, J. Ma, M. Li, X.-H. Li, X.-Q. Dang, and K.-Z. Wang, "Repair effect of coexpression of the $h V E G F$ and $h B M P$ genes via an adeno-associated virus vector in a rabbit model of early steroid-induced avascular necrosis of the femoral head," Translational Research, vol. 166, no. 3, pp. 269-280, 2015.

[32] X.-H. Xie, X.-L. Wang, H.-L. Yang, D.-W. Zhao, and L. Qin, "Steroid-associated osteonecrosis: epidemiology, pathophysiology, animal model, prevention, and potential treatments (an overview)," Journal of Orthopaedic Translation, vol. 3, no. 2, pp. 58-70, 2015.

[33] H. Ding, Y. S. Gao, C. Hu et al., "HIF- $1 \alpha$ transgenic bone marrow cells can promote tissue repair in cases of corticosteroidinduced osteonecrosis of the femoral head in rabbits," PLoS One, vol. 8, no. 5, article e63628, 2013.

[34] Z. Li, W. Liao, X. Cui et al., "Intravenous transplantation of allogeneic bone marrow mesenchymal stem cells and its directional migration to the necrotic femoral head," International Journal of Medical Sciences, vol. 8, no. 1, pp. 74-83, 2011.

[35] P. K. Gupta, A. Chullikana, M. Rengasamy et al., "Efficacy and safety of adult human bone marrow-derived, cultured, pooled, allogeneic mesenchymal stromal cells (Stempeucel ${ }^{\circledR}$ ): preclinical and clinical trial in osteoarthritis of the knee joint," Arthritis Research \& Therapy, vol. 18, no. 1, p. 301, 2016.

[36] P. K. Gupta, A. K. Das, A. Chullikana, and A. S. Majumdar, "Mesenchymal stem cells for cartilage repair in osteoarthritis," Stem Cell Research \& Therapy, vol. 3, no. 4, p. 25, 2012.

[37] Y. Li, J. Xu, L. Mao et al., "Allogeneic mesenchymal stem cell therapy for bisphosphonate-related jaw osteonecrosis in swine," Stem Cells and Development, vol. 22, no. 14, pp. 2047-2056, 2013.

[38] M. D. Griffin, A. E. Ryan, S. Alagesan, P. Lohan, O. Treacy, and T. Ritter, "Anti-donor immune responses elicited by allogeneic mesenchymal stem cells: what have we learned so far?," Immunology \& Cell Biology, vol. 91, no. 1, pp. 40-51, 2013.

[39] J. A. Ankrum, J. F. Ong, and J. M. Karp, "Mesenchymal stem cells: immune evasive, not immune privileged," Nature Biotechnology, vol. 32, no. 3, pp. 252-260, 2014.

[40] M. D. Griffin, T. Ritter, and B. P. Mahon, "Immunological aspects of allogeneic mesenchymal stem cell therapies," Human Gene Therapy, vol. 21, no. 12, pp. 1641-1655, 2010. 


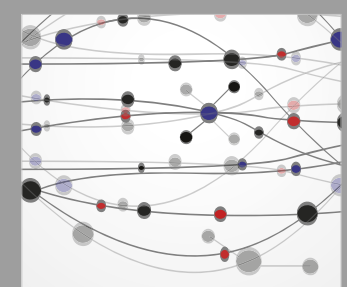

The Scientific World Journal
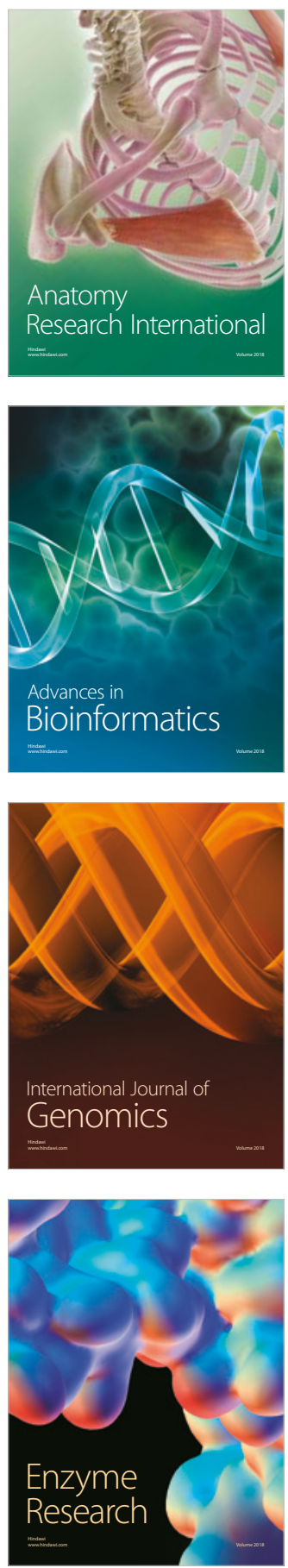
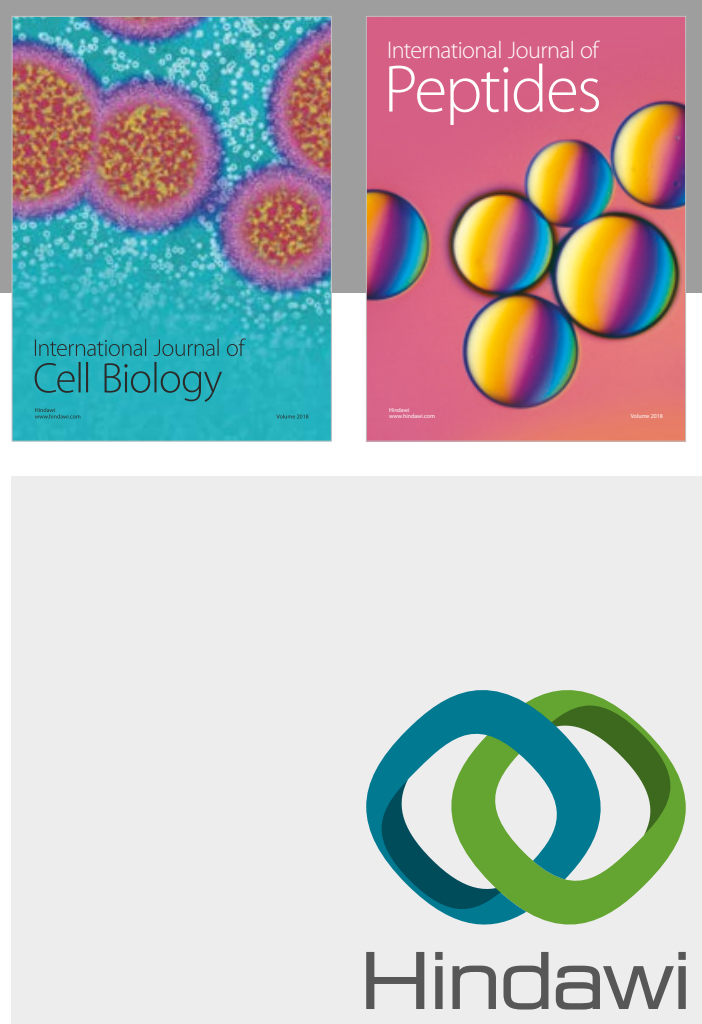

Submit your manuscripts at

www.hindawi.com
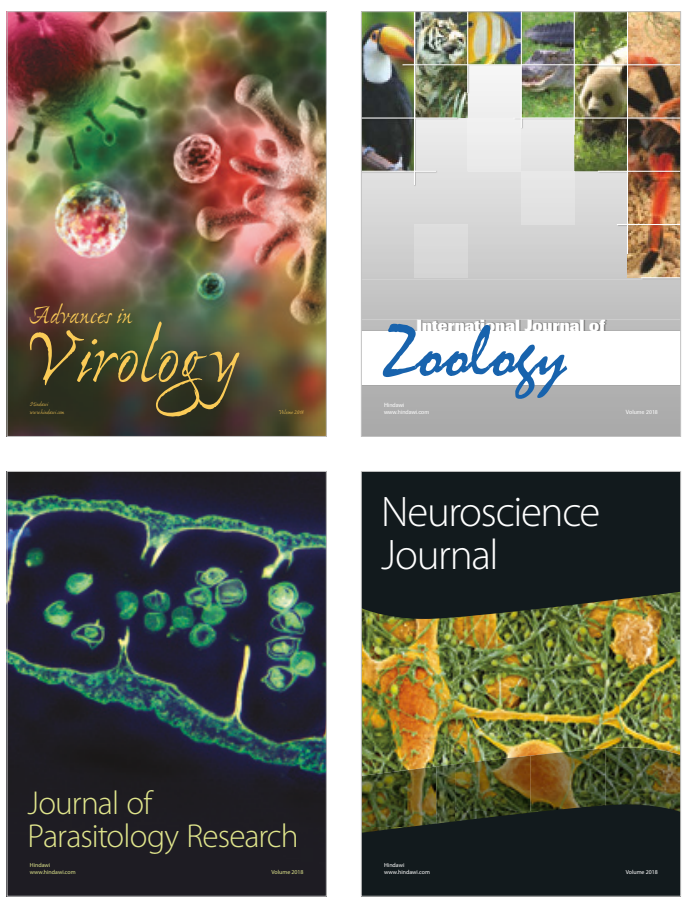
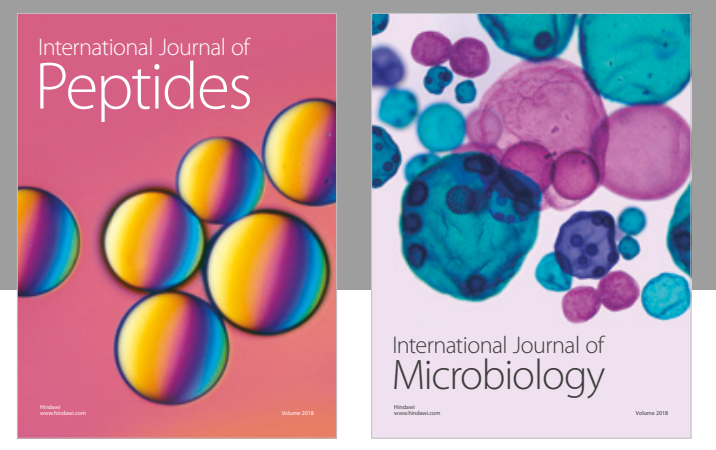

nternational Journal of Microbiology
Journal of
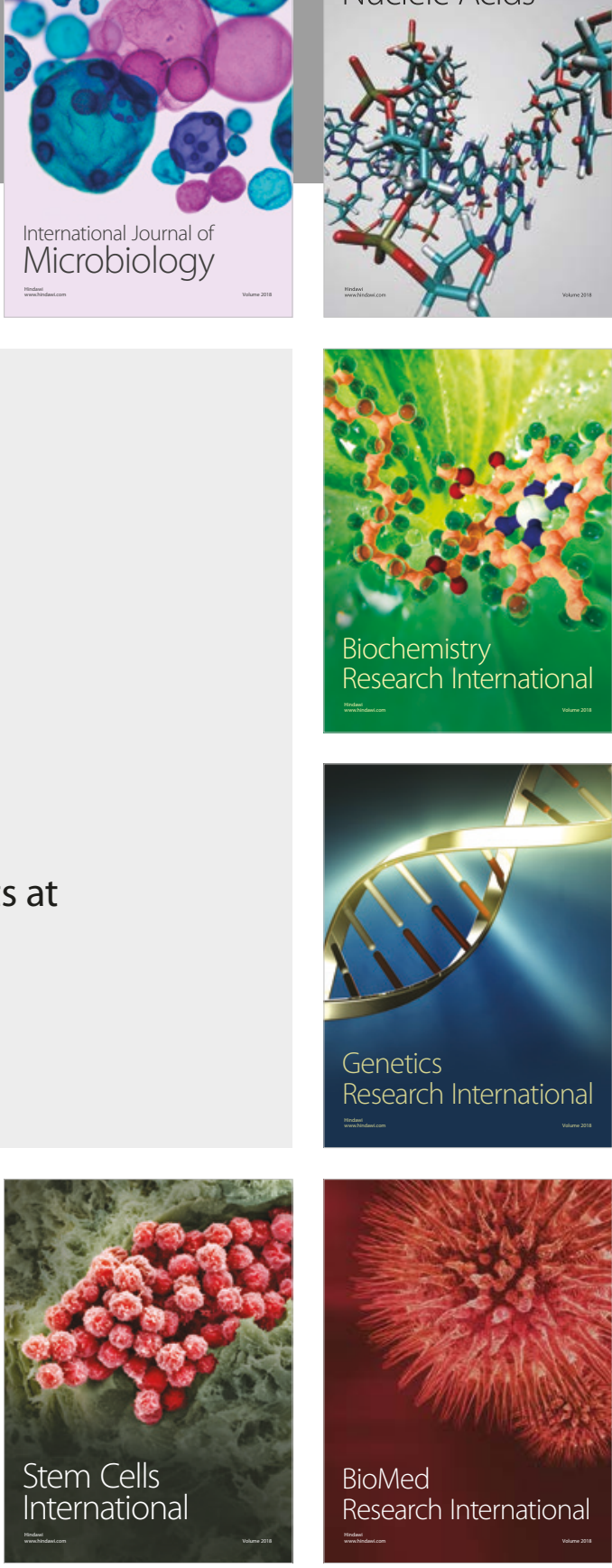
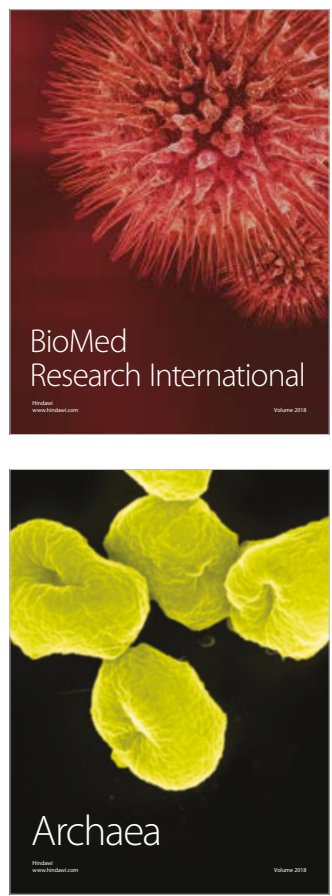